\title{
Short-term memory for serial order supports vocabulary development: New evidence from a novel word learning paradigm
}

\author{
Steve Majerus $^{\mathrm{a}, \mathrm{b}, *}$, Claire Boukebza ${ }^{\mathrm{a}}$ \\ ${ }^{\mathrm{a}}$ Department of Psychology-Cognition and Behavior, Université de Liège, B-4000 Liège, Belgium \\ ${ }^{\mathrm{b}}$ Fonds National de la Recherche Scientifique (FNRS), B-1000 Bruxelles, Belgium
}

\section{A R T I C L E I N F O}

\section{Article history:}

Received 8 February 2013

Revised 24 July 2013

\section{Keywords:}

Short-term memory

Serial order

Vocabulary learning

Lexical development

Serial position effects

Verbal working memory

\begin{abstract}
A B S T R A C T
Although recent studies suggest a strong association between short-term memory (STM) for serial order and lexical development, the precise mechanisms linking the two domains remain to be determined. This study explored the nature of these mechanisms via a microanalysis of performance on serial order STM and novel word learning tasks. In the experiment, 6- and 7-year-old children were administered tasks maximizing STM for either item or serial order information as well as paired-associate learning tasks involving the learning of novel words, visual symbols, or familiar word pair associations. Learning abilities for novel words were specifically predicted by serial order STM abilities. A measure estimating the precision of serial order coding predicted the rate of correct repetitions and the rate of phoneme migration errors during the novel word learning process. In line with recent theoretical accounts, these results suggest that serial order STM supports vocabulary development via ordered and detailed reactivation of the novel phonological sequences that characterize new words.
\end{abstract}

(c) 2013 Elsevier Inc. All rights reserved.

\section{Introduction}

An increasing literature is showing that verbal short-term memory (STM), and especially STM for serial order, is closely associated with lexical development. The underlying hypothesis is that shortterm retention abilities for sequential information support vocabulary development by facilitating

\footnotetext{
* Corresponding author at: Department of Psychology-Cognition and Behavior, Université de Liège, B-4000 Liège, Belgium. E-mail address: smajerus@ulg.ac.be (S. Majerus).
} 
learning of the phonological forms of new words during the initial learning stage, when the novel word form is first encountered. However, evidence for this mainly comes from studies correlating performance on STM tasks with general estimates of receptive vocabulary knowledge that confound phonological, lexical, and semantic levels of knowledge. The aim of this study was to provide direct evidence for a link between serial order STM capacities and learning abilities for novel word forms in children and to gain a deeper insight into the mechanisms that determine this link.

A number of studies have explored the link between verbal STM capacity and vocabulary development, showing a consistent association between estimates of verbal STM, as measured by nonword repetition tasks, and vocabulary knowledge, especially in younger children (e.g., Avons, Wragg, Cupples, \& Lovegrove, 1998; Gathercole, Willis, Baddeley, \& Emslie, 1994; Gathercole, Willis, Emslie, \& Baddeley, 1992; Service, 1992). The difficulty, however, is to clearly understand the factors that drive this association. Typically, a verbal STM task requires immediate repetition of sequences of familiar or unfamiliar verbal information, with the sequences containing either multiple items (e.g., word list immediate serial recall) or single items of variable length (e.g., multisyllabic nonword repetition). In the framework of the phonological loop model, the association between performance on STM tasks and vocabulary development is considered to reflect the importance of temporary phonological storage capacity for forming new long-term phonological lexical representations (e.g., Baddeley, Gathercole, \& Papagno, 1998; Gathercole \& Baddeley, 1989). Studies showing that performance in nonword repetition tasks predicts performance on novel word learning tasks in young children are supportive of this assumption (Gathercole, Hitch, Service, \& Martin, 1997). However, the difficulty here is that verbal STM tasks do not simply reflect the capacity of a specialized STM system but also are, at the same time, influenced by language knowledge. Immediate serial recall tasks using word stimuli lead to higher performance levels than tasks using nonwords, suggesting that lexical knowledge contributes to short-term recall, either indirectly via redintegration processes of the decayed STM trace during retrieval (e.g., Hulme, Maughan, \& Brown, 1991; Schweickert, 1993) or directly via stabilizing feedback activation between language and STM systems during all STM stages (e.g., Baddeley et al., 1998; Martin, Lesch, \& Bartha, 1999). Similarly, at the sublexical level, subtle knowledge about statistical properties of sound co-occurrences for the native language phonology leads to a recall advantage for nonwords containing frequent phonotactic patterns relative to nonwords with less frequent phonotactic patterns (Gathercole, Frankish, Pickering, \& Peaker, 1999; Majerus \& Van der Linden, 2003; Majerus, Van der Linden, Mulder, Meulemans, \& Peters, 2004; Thorn \& Frankish, 2005). Finally, at a semantic level, it has also been shown that semantic knowledge supports immediate serial recall of word lists (Majerus \& D’Argembeau, 2011; Poirier \& Saint-Aubin, 1996; Walker \& Hulme, 1999). This implies that traditional STM tasks reveal at least as much about language processing as they do about STM processing; thus, the association between performance on STM and performance on vocabulary measures could be a by-product of the fact that both measures reflect the level of development of the language system (see also Fowler, 1991; Metsala, 1999).

Therefore, it is important to ensure that the impact of language knowledge on verbal STM tasks is controlled for when studying links between verbal STM and lexical development. It has recently been shown that this can be achieved by distinguishing between item and serial order components in verbal STM tasks. The item component refers to the phonological and lexico-semantic characteristics of the items within a list of memoranda, whereas the serial order component refers to the serial position of the items within the list. Importantly, item recall is known to be affected by lexical and semantic variables, but recall of serial order information is much less affected by these (e.g., Majerus \& D’Argembeau, 2011; Nairne \& Kelley, 2004; Poirier \& Saint-Aubin, 1996; Saint-Aubin \& Poirier, 2005). Similarly, impaired language representations in patients with acquired brain lesion lead to poor item recall but not to poor serial order recall (Attout, Van der Kaa, George, \& Majerus, 2012; Majerus, Norris, \& Patterson, 2007). Finally, neuroimaging studies also show that temporary maintenance of verbal item information actively recruits language processing neural networks in bilateral temporal gyri, whereas temporary maintenance of serial order information recruits a distinct neural network involving the intraparietal sulci (Fiebach, Friederici, Smith, \& Swinney, 2007; Majerus, Poncelet, Van der Linden, et al., 2006; Majerus et al., 2010; Marshuetz, Smith, Jonides, DeGutis, \& Chenevert, 2000). These data suggest that short-term storage of item information involves temporary activation of long-term verbal representations in the language network, whereas this is not the case for 
short-term storage of order information. This is also in line with recent models of verbal STM, which consider that item information is represented via temporary activation within the language network, whereas serial order information is processed by a specialized STM system (Brown, Preece, \& Hulme, 2000; Burgess \& Hitch, 1999, 2006; Gupta, 2006; Majerus \& D’Argembeau, 2011).

Recent studies adopted the item order distinction to explore the association between verbal STM abilities and lexical development. Leclercq and Majerus (2010) designed STM tasks either to maximize processing and retention of serial order information while minimizing item processing requirement (e.g., immediate serial recall of word lists, with the words being sampled from a closed set of highly predictable and familiar items) or to maximize item processing requirements while minimizing serial order processing requirements (e.g., delayed recall of single unfamiliar items such as nonwords challenging the sublexical phonological knowledge system). Using this procedure in a longitudinal study design, the authors were able to show that item and order STM tasks administered in 4-year-old children independently predicted the level of vocabulary knowledge 1 year later, with the order STM measure being the most robust predictor (see also Majerus, Poncelet, Greffe, \& Van der Linden, 2006). Using a different strategy, by distinguishing item and order STM capacities based on the proportion of item and order recall errors in standard immediate serial recall of word lists, Majerus, Heiligenstein, Gautherot, Poncelet, and Van der Linden (2009) further showed that the proportion of order errors correlates with vocabulary knowledge in 6- and 7-year-old children. The theoretical interpretation of these findings is that the ability to temporarily maintain sequence information via a dedicated short-term storage system for order information allows the unfamiliar phoneme sequences that define a novel word to be maintained and replayed in correct order during the learning process, thereby increasing the strength of the new lexical representation being created in the language knowledge base, by considering that the language network (where item representations-phonemes, syllables, and complete word forms-are stored and temporarily activated) and the order maintenance system are strongly interconnected and link to each other (Gupta, 2006; Majerus et al., 2006). Order information is furthermore critical when learning a new word form given that the pool of phonemes in any given language is limited, and consequently it is the sequential ordering of these phonemes that greatly contributes to the distinction between different word forms (e.g., pool vs. loop, lap vs. pal, barrier vs. rabid). Some indirect evidence for this hypothesis stems from earlier studies by Gathercole (1995) showing that repetition of low-wordlike nonwords is a better predictor of later vocabulary knowledge than repetition of high-wordlike nonwords, assuming that serial order processing requirements are greater when repeating nonwords of low wordlikeness. However, low-wordlike and highwordlike nonwords differ not only in serial order processing requirements but also in phonological processing requirements and attentional effort, with low-wordlike nonwords requiring to a larger extent access to sublexical phonological processing and more attentional resources dedicated to phoneme/syllable segmentation and identification processes. Hence, these data are difficult to interpret with respect to the specific role of serial order processing and retention requirements in the association between nonword repetition and vocabulary learning. In sum, except for a general demonstration of an association between performance on serial order STM tasks and estimates of vocabulary knowledge, there is currently no direct and specific evidence for the role of memory for serial order in new word learning.

The aim of this study was to provide a direct test of the hypothesis assuming a link between serial order coding mechanisms in STM and learning of novel phonological word forms in 6- and 7-year-old children and to gain a deeper insight into the mechanisms of this link. STM for serial order was assessed using a serial order reconstruction task, and STM for item information was assessed using a delayed single item repetition task, with both tasks having been used successfully in previous studies to probe order and item STM capacities (Leclercq \& Majerus, 2010; Majerus et al., 2006). To obtain a more direct estimate of serial order coding abilities than in previous studies, we not only used overall task performance on the serial order STM task but also computed an index of the precision of serial order representations based on serial position migration errors produced by the participants in the serial order STM task. In a microanalysis of errors produced during the novel word learning task, this index was then used to determine whether the precision of serial order coding in the STM task predicts the precision of phoneme sequences produced in the novel word learning task, allowing us to test the predictions of the theoretical account linking novel word learning abilities to serial order STM 
abilities in the most direct and precise manner so far. In addition, to control for task-related confounds, STM ability was not only compared with performance on a novel word learning task but also compared with two control learning tasks using familiar words, on the one hand, and visual symbols, on the other. This allowed us to determine the specificity of the relationship between serial order STM abilities and novel word learning while controlling for the role of general learning abilities and attentional abilities shared among the three learning tasks.

\section{The current study}

Order STM was measured via a serial order STM reconstruction task (Leclercq \& Majerus, 2010; Majerus et al., 2006). This task maximized retention capacities for serial order information while minimizing requirements for processing phonological, lexical, and semantic information. It involved the auditory presentation of sequences of animal names by increasing list length. At the end of the list, children received cards depicting the animals that had been presented, and they needed to reconstruct the order of presentation of the animals using the cards. Item retention abilities were minimized because item information was fully provided at recall and only the serial order of the items within the target sequence needed to be reconstructed. The precision of serial order coding was estimated by determining the number of positions each erroneously reconstructed item had migrated and by comparing the number of large migrations (three, four, or five positions) relative to the number of small migrations (one or two positions) (Estes, 1972; Lee \& Estes, 1981); the index was computed in such a way (see Method for details) that a higher value of the index reflected a higher precision of serial coding by assuming that precise serial order representations lead to small distance migrations and broad representations lead to large distance migrations, based on a number of experimental studies showing that the vast majority of migration errors in immediate serial reproduction tasks are one or two position migrations (e.g., Botvinick \& Watanabe, 2007; Burgess \& Hitch, 1999; Henson, 1998). Item STM was assessed via a single nonword delayed repetition task maximizing retention of item information by requiring children to process, store, and repeat short unfamiliar phonological patterns with minimal opportunity for the occurrence of serial order errors (Leclercq \& Majerus, 2010).

The novel word learning task and its two control learning conditions were all paired associate learning tasks and similar to previously used learning procedures by Gathercole and colleagues (1994) and Gupta (2006). To make these learning tasks as naturalistic as possible, the tasks were presented as novel name learning experiments in which the children were introduced, via a story, to unfamiliar individuals (aliens) and were told that the aliens would present themselves and that they needed to learn their names. In the novel word learning condition the names were novel words (unfamiliar word forms respecting English phonotactics), whereas in the word learning control condition they were familiar names (matched on phonological structure to the novel words) and in the visual learning control condition the aliens presented themselves via a visual symbol that was supposed to represent the written forms of their names. Otherwise, the three learning conditions followed exactly the same procedure by including three alien-name pairs to be learned over six successive learning trials. Hence, the only difference among the three conditions was the nature of the items to be learned, with only the novel word learning condition involving learning of a novel sequence of phonemes. The task included three alien-name pairs because pilot testing had shown that this was the optimal number of pairs that could be learned by 6- and 7-year-old children while avoiding floor and ceiling effects in learning performance.

\section{Method}

\section{Participants}

A total of 50 children participated in this study. Their mean age was 79 months $(S D=4.0)$. The children were selected from second-grade classes from different schools in the suburban area of Liege, Belgium. Parental consent was obtained for each child. The parents were also administered a questionnaire ensuring that the children's native language was French, that the children were monolingual, 
that they had no history of neurological disorders or neurodevelopmental delay, that auditory and visual acuity were normal or corrected, and that they had typical language development and no significant learning difficulties. The children lived in families with a middle-class socioeconomic background. They were seen in their respective schools.

\section{Materials and procedure}

Order short-term memory task (animal race task)

This STM task, first validated by Majerus et al. (2006), specifically measured the ability to encode, maintain, and reproduce serial order information in verbal STM. This task has high test-retest reliability (Leclercq \& Majerus, 2010). After the auditory presentation of sequences of animal names (chat, chien, coq, lion, loup, ours, and singe [cat, dog, cock, lion, wolf, bear, and monkey, respectively]), the children needed to rearrange cards depicting the animals as a function of their order of presentation. This task was designed to maximize requirements for processing serial order information. Requirements for processing item information were minimized by selecting highly familiar stimuli of high lexical frequency and low age of acquisition (see Majerus et al., 2006), by selecting stimuli presenting an all monosyllabic structure minimizing phonological processing demands, by making item information known in advance (for all sequences with a length of two, the stimuli cat and lion were used; for all sequences with a length of three, the stimuli cat, lion, and wolf were used; and so forth for the other sequence lengths), and by making item information fully available at recall (cards representing the presented animals were given to the children, who simply needed to arrange them in correct serial position on a staircase with seven steps drawn on a sheet). The seven stimuli were used to form lists with lengths ranging from two to seven items, and there were four trials for each list length. The sequences had been recorded by a female voice at a rate of one item per second, stored on a computer disk, and presented to the children via headphones and by increasing list length. The children were told the following story for task description:

Every year, the animals from all over the world gather to have a huge race. This year, seven animals are participating: a dog, a cat, a lion, a bear, a wolf, a monkey, and a cock [the experimenter shows the cards of the corresponding animals]. Several races take place. Sometimes, only two animals are participating. Sometimes, there are three, four, or five animals. On other times, there are big races with six animals. Through the headphones, you will hear someone announce the animals' order of arrival at the finish line, from the first to the last animal. Immediately after, you have to put the pictures of the animals on the podium in their order of arrival. The animal arriving first has to be put on the highest step and the last one on the lowest step. Okay?

The children were informed when list length increased. As a first global measure of order STM performance, we determined the percentage of correctly placed items by pooling over all sequence lengths as a dependent measure. As a second, more direct measure of the precision of coding and retention of serial order information, we computed a serial position distance effect variable, $D$, for serial order reconstruction errors by comparing the proportion of small position migrations (one or two position migrations relative to the target position) over large position migrations (three to five position migrations relative to the target position). The following formula was used:

$$
D=\frac{\left(D_{1}+D_{2}\right)-\left(D_{3}+D_{4}+D_{5}\right)}{\left(D_{1}+D_{2}\right)+\left(D_{3}+D_{4}+D_{5}\right)}
$$

Migration errors of six positions were not considered because no migration error of this size was observed in any participant.

\section{Item short-term memory task (princess task)}

This STM task, also validated by Majerus et al. (2006), specifically measured the ability to encode, maintain, and reproduce phonological item information in verbal STM. This task has excellent testretest reliability (Leclercq \& Majerus, 2010). The task consisted of 34 monosyllabic nonwords presented separately, and the items were new on any trial. The stimuli had a consonant-vowelconsonant (CVC) syllabic structure and were all legal with respect to French phonotactic rules. 
The diphone frequencies of the CV segments (mean=149, range $=3-524$ ) and VC segments ( mean $=129$, range $=7-728$ ) were chosen to be low relative to the phonological structure of French (Tubach \& Boë, 1990) to maximize the processing demands of phonological item information. By contrast, order information retention was reduced because only a single item needed to be retained. In addition, all nonwords had the same monosyllabic CVC structure, unlike traditional nonword repetition tasks using multisyllabic nonwords of unpredictable syllabic structure. Hence, the only order errors that could occur were inversions between the first and last consonants, which are highly infrequent $(0.4 \%$ of errors in the study by Leclercq \& Majerus, 2010$)$. The nonword stimuli were recorded by a female human voice, stored on a computer disk, and presented via headphones. At the end of each stimulus, the children were instructed to continuously repeat the syllable "bla" for $3 \mathrm{~s}$ and then to repeat the stimulus. Children also needed to repeat the nonword once immediately after presentation to confirm that they had correctly perceived the item and were able to reproduce it accurately. The children were told the following story for task description:

You are an adventurer [for a boy]/a princess [for a girl] locked up in the tower of a castle [a drawing of the castle is shown to the child on the computer screen]. The castle has many doors. You have to find your way out of the castle. In order to do so, you have to open the doors by remembering passwords. More precisely, when you see a closed door [the experimenter shows the next computer slide depicting a closed door], you will hear through the headphones a password which opens the door and which you have to remember. The door opens if you repeat "blablabla" during a short time and if afterward, on my order, you repeat the password you just heard. Okay?

We determined the percentage of phonemes correctly repeated as a dependent variable.

\section{Learning tasks}

The children needed to learn the names of three aliens. In the target nonword condition, they were three unisyllabic nonwords (/riz/, /bam/, /nur); in the word control condition, they were familiar French names matched on phonological structure with the nonword stimuli (/tom/, /lyk/, /3il/); in the visual control condition, they were abstract line drawings symbolizing the written form of the names in the aliens' language (see Fig. 1). For each learning condition, the children saw three differently colored and shaped aliens (see Fig. 1 for examples) who presented their names auditorily (in the two verbal learning conditions) or visually (in the visual learning condition). For each condition, the learning procedure was the following. A first alien appeared on the screen on a background scene depicting a planet, and the prerecorded sentence ("Hello, my name is ...") finishing with the alien's name (in either auditory or visual form, depending on the experimental condition) was presented (total duration of $3 \mathrm{~s}$ for each trial and each condition). Immediately after that, the second alien appeared on the screen, and the sentence ("Hello, my name is ...") finishing with the second alien's name was presented, and so forth for the third alien/name association. After the presentation of the three alien names, a recall procedure was initiated where one of the three aliens reappeared on the screen and the children needed to recall the alien's name, immediately followed by the second and third remaining aliens. Then a new learning trial was initiated, followed by a new recall trial. This procedure was repeated six times. The order of appearance of the three aliens within each learning and recall trial was pseudo-randomized by avoiding exact matches of the alien appearance orders between two adjacent learning and recall trials as well as between two successive learning trials. The children were told the following stories for task description. For the nonword learning condition, the instructions were the following:

You will now meet inhabitants of the planet Mercatus. They want to make your acquaintance, and so they will present themselves to you by telling you their name. You will need to memorize the name and to recall it when the alien reappears. The aliens will tell you this time their names in their own language we do not know. You will never have heard these names before. That's okay. You will have six trials to learn their names. Do you understand? Are you ready?

For the word learning condition, the instructions were the following:

You will now meet other inhabitants of the planet Mercatus. They also want to make your acquaintance, and so they will present themselves to you by telling you their name. You will need to mem- 

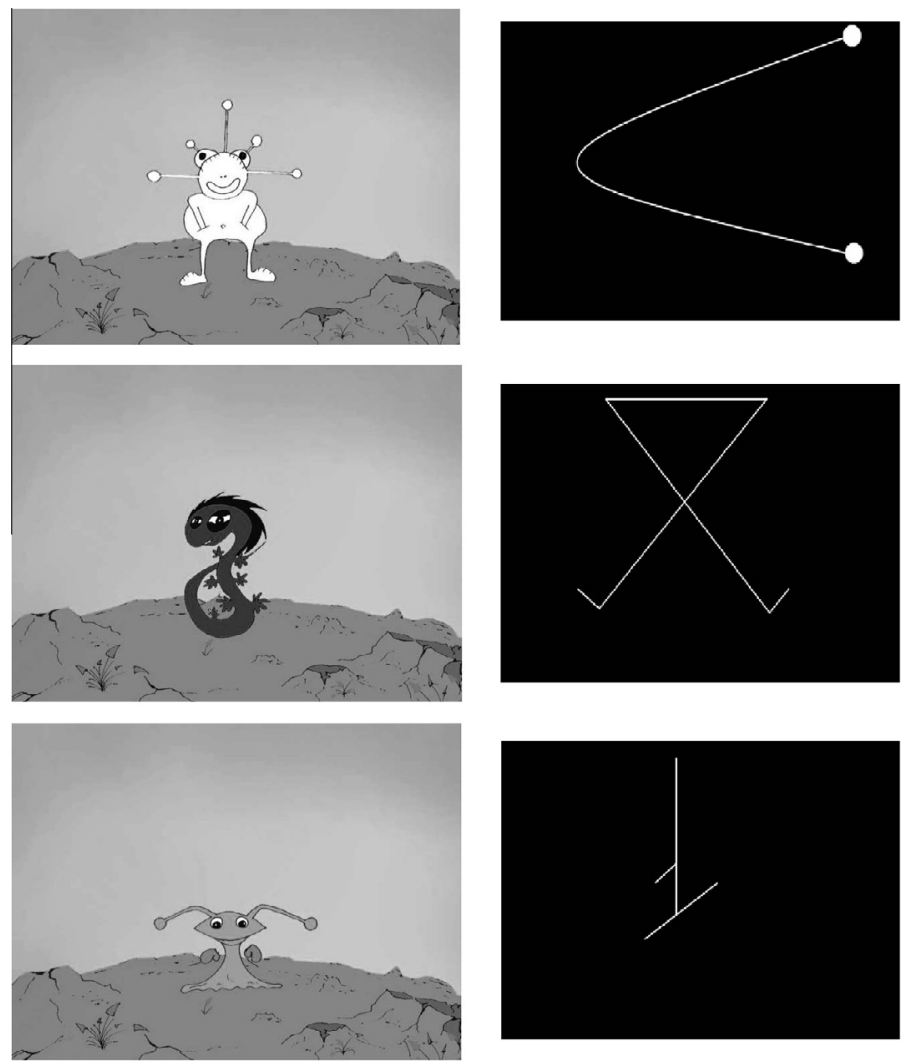

Fig. 1. Examples of the stimuli used in the learning tasks. The left column shows three examples of the object referents (note that the original stimuli were in color), and the right column shows the visual symbols to be learned in the visual symbol learning condition.

orize the name and to recall it when the alien reappears. You will have six trials to learn their names. Do you understand? Are you ready?

In the visual condition, the instructions were the following:

You will now meet other inhabitants of the planet Mercatus. They want to make your acquaintance, and so they will present themselves to you by showing their name written in their alien language. You will need to memorize the name and to draw it on the cards I give you when the alien reappears. You will have six trials to learn their names. Do you understand? Are you ready?

For each learning condition, we computed the number of items correctly recalled over the six learning trials. For ease of reading, the details of the microanalysis of errors produced during the nonword learning task are presented in the Results section.

\section{Receptive vocabulary knowledge}

Receptive vocabulary knowledge was estimated using the standardized EVIP scales (Dunn, Thériault-Whalen, \& Dunn, 1993), a French adaptation of the Peabody Picture Vocabulary Test (Dunn \& Dunn, 1981). As a dependent variable, we used raw vocabulary scores. 
Table 1

Descriptive statistics for all tasks.

\begin{tabular}{lllrr}
\hline & Mean & Standard deviation & Skewness $^{\mathrm{a}}$ & Kurtosis $^{\mathrm{b}}$ \\
\hline EVIP raw score (standard score) & $84.55(116.00)$ & $13.04(11.98)$ & -.23 & -.77 \\
$\quad$ Raven's matrices (standard score) & $23.45(111.51)$ & $4.97(7.86)$ & .43 & -.52 \\
$\quad$ Raven's matrices & & & & \\
$\quad$ Short-term memory & .57 & & .12 & -.41 \\
$\quad$ Serial order & .58 & .13 & -.03 & -.15 \\
$\quad$ Distance effect index & .62 & .15 & -.31 & -.31 \\
$\quad$ Item & .62 & .17 & -.26 & -.33 \\
$\quad$ Learning tasks & .87 & .12 & -1.17 & .55 \\
$\quad$ Novel word & .66 & .16 & -.03 & -.15 \\
$\quad$ Sord & & & & \\
$\quad$ Symbol & & & & \\
\hline
\end{tabular}

Note: All scores reflect the proportions of correct responses if not otherwise specified.

a Standard error Skewness cutoff $= \pm 0.70$.

b Standard error Kurtosis cutoff $= \pm 1.38$.

\section{Nonverbal intelligence}

Raven's Progressive Colored Matrices (Raven, Court, \& Raven, 1998) were administered to control for general intellectual efficiency. Raw scores were used in the analyses presented in the Results section.

\section{Task order}

The different tasks were presented to the children in three sessions, each lasting approximately $25 \mathrm{~min}$. The tasks were presented in pseudo-random order, with one of the three learning tasks and one or two tasks of the remaining set of tasks per session. To avoid fatigue and stimulus interference effects biasing learning performance, learning tasks were always presented first in each session.

\section{Results}

The data from 47 children were retained for further analysis; the data from 3 children had been excluded from analysis due to outlier performance on the receptive vocabulary measure (1 case) or logistic problems during task administration (2 cases). Descriptive statistics are shown in Table 1. An examination of the distribution of scores showed that for all measures, Skewness and Kurtosis estimates remained within the recommended range of 2 standard errors except for the word learning condition (Tabachnick \& Fidell, 1996); this was related to mean performance being overall closer to the maximum score, which was expected for this control learning task where familiar information needed

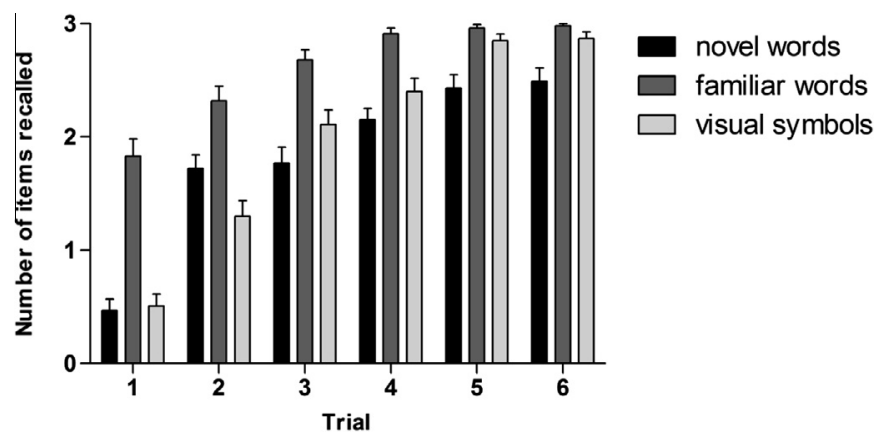

Fig. 2. Performance on the learning tasks as a function of learning trial. 


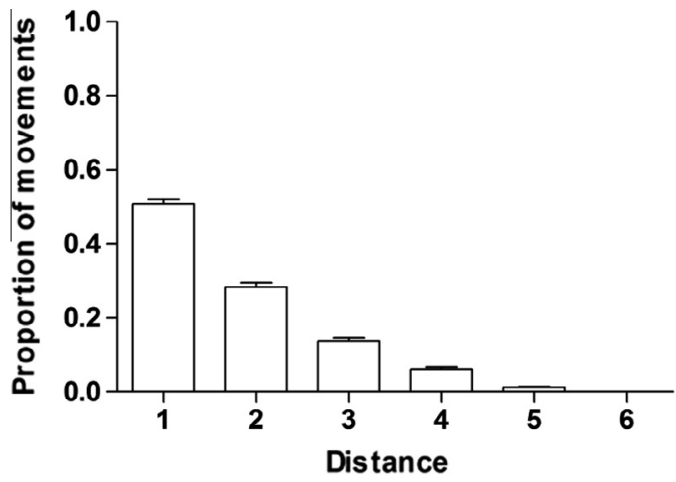

Fig. 3. Serial position movement gradients for the serial order STM task.

to be learned. This is also indicated by the results of learning performance as a function of learning trials, as shown in Fig. 2; for the familiar word learning condition, the three object-word associations were mastered on average after the second learning trial, whereas the maximum of learning performance was reached only on the final learning trials for the novel word and visual symbol learning conditions. It is also important to note that the item and order STM conditions led to nearly identical mean levels of performance, indicating that task difficulty was balanced across tasks. The same was also true for the novel word and visual symbol learning conditions, which showed comparable means and standard deviations and a similar progression of learning over the different learning trials. Finally, as expected, the mean value of the distance effect index was positive, showing that overall the proportion of short distance migration errors in the serial order STM task was higher than the proportion of long distance migration errors. This is further illustrated in Fig. 3, showing that the majority of migration errors involved one or two serial position movements, in line with previous studies on serial position gradients in serial recall tasks (e.g., Estes, 1972; Lee \& Estes, 1981; McCormack, Brown, Vousden, \& Henson, 2000).

\section{Correlation analyses}

All correlations presented in the following section are partial correlations controlling for the effect of age. A first set of correlation analyses assessed the overall relationship between the learning measures and all other measures. As shown in Table 2, both the serial order STM score and the distance effect index correlated significantly with the novel word learning measure. These results indicate that it is not only overall performance on the serial order STM tasks that predicts novel word learning ability, but also more directly the precision of serial order coding and maintenance that is measured by the distance effect index. This index is also not simply the consequence of higher task performance that would automatically lead to a higher proportion of large distance migration errors because the correlation between the distance effect index and serial order STM performance, although significant, was far from reflecting a perfect association. This is also in line with the fact that performance on the item STM task correlated significantly with overall performance on the serial order STM task but not with the distance effect index, reflecting the precision of serial order coding; however, note that the 95\% confidence intervals of the two correlations are partially overlapping. Finally, word learning performance was significantly associated with general vocabulary knowledge and the item STM task. This is consistent with the dependence of the word learning condition, which involves processing of familiar words, on the recruitment of existing lexico-semantic representations, of which the vocabulary task is also a reflection. The association between the word learning task and the item STM task may also reflect this common dependence on the activation and quality of the underlying language representations, but the correlation needs to be considered with caution given the deviation from normality of the distribution of the word learning scores. The visual symbol learning task was also associated 
Table 2

Correlations between learning task and all other tasks (after controlling for age) and 95\% confidence intervals.

\begin{tabular}{|c|c|c|c|c|c|c|c|}
\hline & EVIP & Order STM (score) & Order STM (distance effect) & Item STM & Novel word learning & Word learning & Symbol learning \\
\hline Raven's matrices & $\begin{array}{l}.22 \\
(-.09 \text { to } .54)\end{array}$ & $\begin{array}{l}.26 \\
(-.03 \text { to } .54)\end{array}$ & $\begin{array}{l}.16 \\
(-.10 \text { to } .40)\end{array}$ & $\begin{array}{l}.25 \\
(-.06 \text { to } .51)\end{array}$ & $\begin{array}{l}.23 \\
(.00 \text { to } .44)\end{array}$ & $\begin{array}{l}.04 \\
(-.26 \text { to } .30)\end{array}$ & $\begin{array}{l}.37^{*} \\
(.05 \text { to } .67)\end{array}$ \\
\hline EVIP & - & $\begin{array}{l}.28^{(*)} \\
(.00 \text { to } .51)\end{array}$ & $\begin{array}{l}.11 \\
(-.17 \text { to } .39)\end{array}$ & $\begin{array}{l}.14 \\
(-.16 \text { to } .43)\end{array}$ & $\begin{array}{l}.27 \\
(.03 \text { to } .49)\end{array}$ & $\begin{array}{l}.47^{* *} \\
(.19 \text { to } .68)\end{array}$ & $\begin{array}{l}.13 \\
(-.12 \text { to } .39)\end{array}$ \\
\hline Order STM (score) & & - & $\begin{array}{l}.38^{* *} \\
(.05 \text { to } .66)\end{array}$ & $\begin{array}{l}.32^{*} \\
(.11 \text { to } .52)\end{array}$ & $\begin{array}{l}.50^{* *} \\
(.29 \text { to } .67)\end{array}$ & $\begin{array}{l}.26 \\
(.02 \text { to } .48)\end{array}$ & $\begin{array}{l}.18 \\
(-.09 \text { to } .45)\end{array}$ \\
\hline Order STM (distance effect) & & & - & $\begin{array}{l}-.03 \\
(-.36 \text { to } .35)\end{array}$ & $.33^{*}(-.03$ to .63$)$ & $\begin{array}{l}.04 \\
(-.27 \text { to } .35)\end{array}$ & $\begin{array}{l}.10 \\
(-.15 \text { to } .32)\end{array}$ \\
\hline Item STM & & & & - & $\begin{array}{l}.19 \\
(-.10 \text { to } .48)\end{array}$ & $\begin{array}{l}.33^{*} \\
(.04 \text { to } .56)\end{array}$ & $\begin{array}{l}.14 \\
(-.18 \text { to } .41)\end{array}$ \\
\hline Novel word learning & & & & & - & $\begin{array}{l}.21 \\
(-.07 \text { to } .45)\end{array}$ & $\begin{array}{l}.34^{*} \\
(.12 \text { to } .56)\end{array}$ \\
\hline Word learning & & & & & & - & $\begin{array}{l}.10 \\
(-.17 \text { to } .35)\end{array}$ \\
\hline
\end{tabular}

Note: The 95\% confidence intervals, estimated by a bootstrap procedure with 1000 samples (Efron \& Tibshirani, 1993; Thompson, 1993), are in parentheses.

${ }^{(*)} p=.06$.

${ }^{*} p<.05$.

$p<.01$ 
Table 3

Multiple hierarchical regression analyses for predictor variables of novel word learning condition.

\begin{tabular}{lllllllrl}
\hline Variable introduced & $\Delta R^{2}$ & $B$ & $S E(B)$ & Beta & \multicolumn{1}{l}{$p$} & \multicolumn{1}{l}{$F$} & \multicolumn{1}{l}{$p$} \\
\hline 1. Raven's matrices & .06 & 0.16 & 0.06 & .24 & .10 & 2.82 & .10 & 1,45 \\
2. EVIP & .06 & 0.06 & 0.04 & .26 & .08 & 3.14 & .05 & 2,44 \\
3. Word learning & .01 & 0.17 & 0.24 & .11 & .50 & 2.22 & .09 & 3,43 \\
4. Symbol learning & .07 & 0.30 & 0.16 & .29 & .06 & 2.68 & $<.05$ & 4,42 \\
5. Item STM & .01 & 0.09 & 0.25 & .09 & .57 & 2.18 & .08 & 5,41 \\
6. Order STM (score) & .15 & 0.14 & 0.05 & .42 & $<.01$ & 3.60 & $<.01$ & 6,40 \\
7. Order STM (distance effect) & .02 & 3.99 & 3.59 & .16 & .27 & 3.28 & $<.01$ & 7,39 \\
8. Order STM (distance effect) & .08 & 7.16 & 3.49 & .28 & $<.05$ & 2.66 & $<.05$ & 6,40 \\
9. Order STM (score) & .09 & 0.12 & 0.05 & .35 & $<.05$ & 3.28 & $<.01$ & 7,39 \\
\hline
\end{tabular}

Note: To avoid over-parameterization, the variable age was not included in these multiple regression analyses; when we added the variable age in a follow-up analysis, the total amount of variance explained increased by less than $1 \%$ and did not alter the significance of the other predictor variables.

with performance on Raven's matrices, which is consistent with the shared involvement of visuo-spatial processing abilities in both tasks. Finally, the vocabulary knowledge scores also showed a marginally significant correlation with performance on the serial order STM task, in line with previous studies.

\section{Regression analyses}

The next analysis determined the specificity of the association between serial order STM abilities and novel word learning via a set of multiple hierarchical regression analyses. These analyses controlled for nonverbal intellectual efficiency, vocabulary knowledge, general task-related learning processes such as attentional focalization shared with the word and symbol learning tasks, and general STM processes shared with the item STM task; although most of these variables did not show a significant correlation with novel word learning, they may yet partially explain the association between serial order STM abilities and novel word learning, as indicated by the overlapping confidence intervals of correlations reported in Table 2. We successively introduced Raven's matrices, vocabulary, word learning, symbol learning and item STM scores, and then we introduced the serial order STM performance score and the serial order STM distance effect index. When introducing the serial order STM distance effect index before the general serial order STM score, this index independently predicted novel word learning performance (see Table 3 ). When introducing the general serial order STM score before the distance effect index, the distance effect index provided no independent prediction of novel word learning anymore, whereas the general serial order STM score still did. These data show that performance on the serial order STM strongly and specifically predicts novel word learning performance after controlling for general processes such as nonverbal intellectual efficiency, existing vocabulary knowledge, task-related attentional processes, and item STM capacities. Furthermore, a more precise measure of serial order coding, the serial order STM distance effect index, also predicts novel word learning performance, but only before the introduction of the serial order STM score, suggesting that its predictive power is tied to the general serial order STM performance score. This was expected because the serial order STM distance effect index isolates one specific factor among the factors contributing to overall performance on the serial order STM reconstruction task. The specific role of the serial order STM distance effect index in nonword learning is explored more directly in the next section.

\section{Microanalysis of novel word learning task}

Next, we aimed at determining the mechanisms driving the association between the novel word learning task and serial order STM ability. First, we computed a learning speed variable that reflects the speed at which all three novel words were acquired. If the association between serial order STM variables and performance on the novel word learning task really means that serial order STM supports novel word learning per se, then serial order STM should be associated not only with overall 
Table 4

Descriptive statistics for task microanalysis variables of novel word learning task.

\begin{tabular}{lrrrr}
\hline & Mean & Standard deviation & Skewness $^{\mathrm{a}}$ & Kurtosis $^{\mathrm{b}}$ \\
\hline Learning speed index & 5.26 & 1.67 & -.66 & -.47 \\
Omission error proportion & .21 & .16 & .73 & -.41 \\
Phoneme migration error proportion & .13 & .11 & -.63 & -.36 \\
Other errors (proportion) & .05 & .06 & 1.38 & 1.25 \\
Phoneme migration score $^{\mathrm{c}}$ & -0.01 & 0.52 & 0.20 & -0.47 \\
\hline
\end{tabular}

a Standard error skewness cutoff $= \pm 0.69$.

b Standard error kurtosis cutoff $= \pm 1$.36.

c $N=36$.

performance on the novel word learning task but also with variables reflecting the novel word learning process more directly. We computed a learning index that represents novel word learning speed and reflects the earliest trial number at which learning was stabilized. That is, all three novel words were correctly recalled on that trial and all subsequent trials; if the learning criterion was not reached by the sixth trial, then a score of 7 was allocated, leading to a learning speed index varying between 1 and 7. Second, we performed a microanalysis of errors produced on the novel word learning task by considering omission errors (no output is produced), phoneme migration errors that at the same time can be assimilated to partially correct responses (the response produced for a given target is a recombination of the phonemes of the target novel word and of the other novel words), and other error types (phoneme intrusion errors [i.e., phonemes do not occur in any of the target novel words] and pairing errors [i.e., the novel nonword is correct but produced with the wrong cue word]). If serial order STM supports vocabulary learning via ordered replay of novel phoneme sequences, as discussed in the Introduction, then serial order STM ability should be inversely related to the amount of omission errors but should also predict the amount of phoneme migration errors during novel phoneme sequence reproduction.

The descriptive statistics of the learning speed index and the different error types are presented in Table 4. The mean value for the learning speed index was 5.26, meaning that on average participants had acquired the three target novel words by the fifth trial. For error types, omission errors and phoneme migration errors were the predominant error types, whereas the "other errors" category led to a very low error frequency (less than one error on average) despite the fact that this category already combined two error types (phoneme intrusion and pairing errors). This also shows that this learning task is a sensitive measure of phonological sequence learning because the vast majority of errors, after omission errors, were partially correct responses with phonological sequencing errors. The following analyses focused on the omission and phoneme migration error types as well as the learning speed index. For phoneme migration errors, a phoneme migration score was further computed by proportionalizing phoneme migration errors relative to the omission errors using the formula

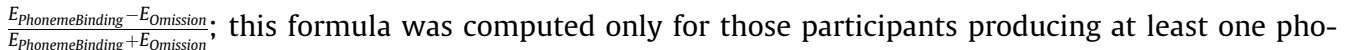
neme migration error $(n=36)$ in order to take into account the possibility that some participants may have been aware of these errors and thus may have produced no output at all, leading to an inflation of omission errors and a wrong estimation of the real frequency of phoneme migration errors. The correlations between the different STM measures, the learning speed index, and the error types are presented in Table 5. In line with previous results, on the one hand, the item STM task did not significantly correlate with any of the micro-measures of the novel word learning task. ${ }^{1}$ On the other hand, both the serial order STM score and the serial order distance effect index correlated significantly with the learning speed index, showing that serial order STM abilities not only are associated with overall repetition performance on a novel word learning task but also actually predict the learning rate of novel words. For the error analyses, a specific profile emerged with respect to omission and phoneme migra-

\footnotetext{
${ }^{1}$ It should also be noted here that although the stimuli used in the item STM task were structurally very similar to the stimuli used in the nonword learning task, this type of phonological sequencing error was very rare in that task because only a single item needed to be maintained per trial (proportion of phoneme migration errors $=.09$ ). This type of error was more than three times more frequent in the novel word learning task (proportion of phoneme migration errors $=.34$ ).
} 
Table 5

Correlations between task microanalysis variables of novel word learning task and short-term memory predictor variables (after controlling for age) and 95\% confidence intervals.

\begin{tabular}{llll}
\hline & Item STM & Order STM (score) & Order STM (distance effect) \\
\hline Learning speed & -0.09 & $-.39^{* *}$ & $-.29^{*}$ \\
& $(-.45$ to .25$)$ & $(-.60$ to -.15$)$ & $(-.58$ to .09$)$ \\
Omission errors & -0.06 & $-.50^{* *}$ & $-.27^{(*)}$ \\
& $(-.36$ to .21$)$ & $(-.67$ to -.29$)$ & $(-.54$ to .03$)$ \\
Phoneme migration score & -.19 & .14 & $.35^{*}$ \\
& $(-.49$ to .17$)$ & $(-.14$ to .45$)$ & $(.06$ to .60$)$ \\
\hline
\end{tabular}

Note: The 95\% confidence intervals, estimated by a bootstrap procedure with 1000 samples (Efron \& Tibshirani, 1993; Thompson, 1993), are in parentheses.

(*) $p=.066$.

${ }^{*} p<.05$.

$p<.01$.

tion scores. The phoneme migration score correlated positively with serial order STM performance, most clearly with the distance effect measure. The omission score correlated negatively with serial order STM performance, most strongly with the overall serial order STM performance score. All of the correlations with phoneme migration errors versus those with omission errors differed significantly $(p<.001)$. When comparing the correlations with error types for the item and order STM tasks, the correlations with omission errors also differed significantly for the item STM score and the serial order STM score $(p<.05)$. Likewise, the correlations with the phoneme migration score significantly differed for the item STM score and the serial order STM distance effect measure $(p<.05)$.

In other words, participants with the best overall serial order STM performance produced fewer omission errors. At the same time, participants with the highest precision of serial order representation (as reflected by the distance effect index) also produced more phoneme migration errors. At first sight, the latter result might seem paradoxical because one could have expected that better precision of serial order representations leads to more precise representation of novel phoneme sequences in a word learning task and, hence, to lower phoneme migration errors. Two elements need to be considered here. First, participants with higher abilities to represent serial order information in STM will be able to repeat a higher number of correct phoneme sequences, as already shown in the preceding section. Second, given that they will be able to represent more detailed sequences and a higher number of them, this will also increase the likelihood that they will commit phoneme migration errors because more target phonemes are maintained and linked within serial order STM. At the same time, these migration errors are also partially correct responses because they share at least one phoneme with the correct response; hence, relative to participants who will produce no output at all or an output differing to a larger extent from the target (e.g., intrusion errors), part of the correct representation for the target novel word is already formed and the complete representation will be learned faster because only the erroneous phoneme-position associations need to be updated. In other words, phoneme migration errors, relative to omission errors, also represent more frequent attempts to produce the target novel word during the learning process and eventually will lead to faster learning. If this is true, then the occurrence of phoneme migration errors should actually be indicative of the ongoing learning process. We checked these predictions by correlating omission errors and the phoneme migration error score with the learning speed index; a strong positive correlation was observed between the learning speed index and omission errors $\left(r=.58, C_{95 \%}=.41\right.$ to $\left..74, p<.001\right)$, showing that the higher the number of omission errors, the slower the learning process, and a negative but nonsignificant correlation was observed when correlating the phoneme binding error score with the learning speed index $\left(r=-.24, C_{95 \%}=-.51\right.$ to $\left..06, p=.16\right)$, showing that participants with a higher proportion of phoneme migration errors tended to show a faster learning process.

\section{Discussion}

The aim of this study was to gain deeper insights into the nature and mechanisms of the link associating serial order STM capacity and vocabulary development. By controlling for general processes 
involved in learning tasks, multiple hierarchical regression analyses showed that serial order STM measures independently predicted novel word learning abilities. Furthermore, we showed that it is not only the overall ability to store serial order information that predicts novel word learning abilities but also, importantly, there is a direct relation between the precision of serial order coding in STM and the rate of phoneme migration errors during novel word learning, providing the most direct evidence so far for an involvement of serial order STM mechanisms in novel word learning.

Relative to previous studies addressing the relationships between STM and vocabulary development, the current study leads to several new findings. First, this study is the first to directly show that serial order STM capacities are a critical component of vocabulary learning in children. This finding is important because it shows in an unambiguous way that the link between verbal STM capacities and vocabulary is not simply mediated by the level of development of the language network that supports both verbal STM and vocabulary tasks. Previous studies could not answer this question because the STM tasks that were used, such as multisyllabic nonword repetition tasks, were very strongly dependent on phonological segmentation and sublexical representation capacities while at the same time confounding item and order processing components (e.g., Gathercole et al., 1994, 1997). Recent studies distinguishing between item and order information were able to show that the serial order component of STM was a critical determinant of receptive vocabulary knowledge as measured by general vocabulary measures (Leclercq \& Majerus, 2010; Majerus et al., 2006, 2009). These studies, however, did not directly show that this association was due to serial order STM actually supporting learning of novel word forms during the vocabulary acquisition process. Two studies had investigated the latter question in adult populations, showing that serial order STM capacity predicted performance on a novel word learning task but without controlling for a potential confound with general learning mechanisms (Majerus, Poncelet, Elsen, \& Van der Linden, 2006; Majerus, Poncelet, Van der Linden, \& Weekes, 2008). We showed in this study that serial order STM abilities specifically support learning of the novel phonological forms that characterize new words in children after controlling for learning abilities of semantic referent-word form associations and object-visual symbol associations. Importantly, this study is the first to show that it is not only the overall ability to store serial order information in STM that supports the novel word learning process, but also the precision with which serial order representations can be maintained in STM, by demonstrating that the distance effect index as an estimate of serial order coding precision predicts the speed of novel word learning as well as the amount of phoneme sequence errors during the learning process. Hence, this study provides the most direct evidence so far for the importance of temporary serial order representation and maintenance mechanisms during the learning process of new phonological sequences that characterize novel words.

It could be argued that the link between serial order STM and novel word learning, relative to the item STM task, is due to general task-related factors such as rehearsal. Rehearsal was blocked in the item STM task, whereas serial rehearsal was possible in the serial order reconstruction task. This was a deliberate methodological choice because the theoretical account proposed here associates serial order STM and novel word learning not only via the precision of phoneme order codes but also via the sequential repetition of these codes (see also below); the item STM task was precisely designed not to tap these processes in order to test the theoretical account proposed here. It follows that we may expect that item STM tasks present a stronger correlation with novel word learning if rehearsal is allowed. It should be argued here that item-based rehearsal was in fact encouraged at least during stimulus encoding because participants needed to repeat the nonword once immediately after presentation in order to check for accurate stimulus encoding. Furthermore, studies using a different task setup and estimating item and order STM abilities based on the proportion of item and order errors in an item and serial order reconstruction task, and allowing for both item-based and order-based rehearsal, yielded similar results as those obtained here, with only order errors showing a specific association with existing vocabulary knowledge (Majerus et al., 2009). However, it remains to be shown whether the same would apply when relating this type of item and order reconstruction task to the novel word learning tasks used in this study. Importantly, the association observed here between the distance effect index and novel word learning shows that it is not only serial rehearsal that accounts for the association between performance on the serial order STM task and novel word learning but also the precision at which serial order information is coded and rehearsed. 
At a theoretical level, models relating novel word learning to verbal STM capacities remain relatively vague with respect to the exact processes involved (e.g., Baddeley et al., 1998; Majerus et al., 2009). Connectionist models have been proposed, considering that the learning of novel words shares many processes with short-term reproduction of novel phonological forms. Gupta (2006) and Burgess and Hitch $(1999,2006)$ proposed that the serial order representations are maintained via a specific serial order processing system (a positional buffer in the model proposed by Gupta and a dynamic context module in the model proposed by Burgess and Hitch) that is connected to the phonological language network, allowing the model to encode at which position or at which time a given phoneme has been activated in the phonological system. The sequential representations held in the serial order processing system will enable the replay and reactivation of the newly activated phoneme sequences in the phonological system. By replaying the phoneme sequences in correct serial order several times, the phonemes will progressively get bound together in a new long-term memory representation via slow-changing Hebbian adjustments among phonological, lexical, and semantic levels of representation (see also related accounts by, Brown \& Hulme, 1996; Page \& Norris, 2009 and Szmalec, Duyck, Vandierendonck, Mata, \& Page, 2009, which share the same general assumptions about novel word learning mechanisms). The more a novel phonological sequence is repeated, the faster it will get transformed into a stable new word form. The current data provide the most direct evidence so far for this theoretical account of novel word learning. We showed that higher capacities to store serial order information are associated with faster learning and a higher number of entirely correct novel word form repetitions during the learning process. Furthermore, the precision at which serial order information can be represented is specifically associated with the amount of phoneme migration errors during the learning process; participants with higher precision not only produced more entirely correct phoneme sequences but also produced more partially correct phoneme sequences as a result of phoneme recombinations between the target and other novel words. Thus, participants with more developed and precise abilities to store serial order information are able to attempt producing the novel word form more often for a fixed number of learning trials even if the result may be only partially correct during the first repetition attempts. The partially correct responses show that the learning process has been initiated, and the phoneme migration errors show that a finely segmented representation of the target phonological sequences has occurred, although all phonemes are not yet linked to their correct serial position. Hence, learning will be faster than it is for participants providing no attempt at all at producing the novel word form; as we have seen, the number of total or partial replays of the target phoneme sequence in the phonological network will be a determinant for the learning process. This is also supported by recent lexical learning studies showing that Hebb-like sequential repetition learning appears to be a critical component of learning of lexical forms (Szmalec et al., 2009). Finally, this account is not in contradiction with previous accounts based on the phonological loop model but rather extends and refines them. Ellis and Sinclair (1996) and Papagno, Valentine, and Baddeley (1991) showed that by blocking the articulatory rehearsal process, novel word learning abilities in adults are disturbed, arguing for the importance of phonological rehearsal during the learning process. This interpretation is in agreement with the account developed here by stressing again the importance of sequence repetition in novel word form learning; however, the current data show that it is more specifically the serial order processing component of phonological STM that is critical for ensuring ordered repetition of the new phonological sequences.

These results have implications not only for the understanding of the relationship between verbal STM and lexical language development but also for other domains where learning of sequential information and structure is critical. This is, for example, the case for reading acquisition, where sequential graphemic inputs need to be mapped to their corresponding phonological output, especially in novice readers or when reading unfamiliar words. Recent studies have indeed shown that serial order STM predicts written language acquisition (Martinez Perez, Majerus, \& Poncelet, 2012a), and sequential memory more generally appears to be impaired in children and adults, presenting difficulties in written language acquisition (Martinez Perez, Majerus, \& Poncelet, 2012b, 2013; Szmalec, Loncke, Page, \& Duyck, 2011). Number processing is a further domain where sequential processes and the maintenance of sequential information may be critical, as suggested by recent studies indicating a close association between developmental dyscalculia and ordinal processing of numerical information (Kaufman, Vogel, Starke, Kremser, \& Schocke, 2009). 
Finally, this study also suggests that the link between STM and new word learning cannot be accounted for only by general factors such as focused attention capacities. A number of studies have shown that "passive" verbal STM tasks, such as those used in this study, are determined by participants' ability to keep the memoranda in the focus of attention, especially during encoding (Cowan, Fristoe, Elliott, Brunner, \& Saults, 2006; Cowan et al., 2005; Majerus et al., 2009, 2012). These attentional abilities are also likely to be critical during novel word learning, where learners must direct their attentional focus to the novel word being presented. We indirectly controlled for this possibility by administering a visual symbol learning task of similar task difficulty as the novel word learning task and recruiting focused attention processes to a similar extent. Yet, the association between serial order STM abilities and the learning tasks was significant only for the novel word learning condition, indicating that general attentional factors cannot solely explain the observed association between STM and novel word learning measures.

To conclude, the current study provides new insights into the nature of the relationship between verbal STM and novel word acquisition by providing direct evidence for the importance of the serial order processing component of STM tasks. The results also provide strong empirical support for recent theoretical models of STM and verbal learning that argue for a close interaction between a dedicated serial order processing component and the language network and allow us to obtain a more precise picture of the nature of the mechanisms that govern these interactions.

\section{Acknowledgments}

This study was supported by an Inter-University Attraction Pole (IUAP) grant from the Belgian Federal Science Policy Administration (IUAP P7/11). We thank C. Elhorga for help in stimulus construction, and all of the children, parents, and teachers for their collaboration and time devoted to the study.

\section{References}

Attout, L., Van der Kaa, M. A., George, M., \& Majerus, S. (2012). Distinguishing verbal short-term memory and language impairment: The importance of short-term memory for serial order. Aphasiology, 26, 355-382.

Avons, S. E., Wragg, C. A., Cupples, L., \& Lovegrove, W. J. (1998). Measures of phonological short-term memory and their relationship to vocabulary development. Applied Psycholinguistics, 19, 583-601.

Baddeley, A., Gathercole, S., \& Papagno, C. (1998). The phonological loop as a language learning device. Psychological Review, 105, $158-173$.

Botvinick, M., \& Watanabe, T. (2007). From numerosity to ordinal rank: A gain-field model of serial order representation in cortical working memory. Journal of Neuroscience, 27, 8636-8642.

Brown, G. D. A., \& Hulme, C. (1996). Nonword repetition, STM, and age-of-acquisition: A computational model. In S. E. Gathercole (Ed.), Models of short-term memory (pp. 129-148). Hove, UK: Psychology Press.

Brown, G. D. A., Preece, T., \& Hulme, C. (2000). Oscillator-based memory for serial order. Psychological Review, 107, $127-181$.

Burgess, N., \& Hitch, G. J. (1999). Memory for serial order: A network model of the phonological loop and its timing. Psychological Review, 106, 551-581.

Burgess, N., \& Hitch, G. J. (2006). A revised model of short-term memory and long-term learning of verbal sequences. Journal of Memory and Language, 55, 627-652.

Cowan, N., Elliott, E. M., Saults, J. S., Morey, C. C., Mattox, S., Hismjatullina, A., et al (2005). On the capacity of attention: Its estimation and its role in working memory and cognitive aptitudes. Cognitive Psychology, 51, 42-100.

Cowan, N., Fristoe, N. M., Elliott, E. M., Brunner, R. P., \& Saults, J. S. (2006). Scope of attention, control of attention, and intelligence in children and adults. Memory \& Cognition, 34, 1754-1768.

Dunn, L. M., \& Dunn, L. M. (1981). Peabody picture vocabulary test-revised: Manual for forms L and M. Circle Pines, MN: American Guidance Service.

Dunn, L. M., Thériault-Whalen, C. M., \& Dunn, L. M. (1993). Echelle de vocabulaire en images peabody: Adaptation française du peabody picture vocabulary test. Toronto, ON, Canada: PsyCan.

Efron, B., \& Tibshirani, R. J. (1993). An introduction to the bootstrap. New York: Chapman \& Hall.

Ellis, N. C., \& Sinclair, S. G. (1996). Working memory in the acquisition of vocabulary and syntax: Putting language in good order. Quarterly Journal of Experimental Psychology A, 49, 234-250.

Estes, W. K. (1972). An associative basis for coding and organization in memory. In A. W. Melton \& E. Martin (Eds.), Coding processes in human memory. Washington, DC: V. H. Winston.

Fiebach, C. J., Friederici, A. D., Smith, E. E., \& Swinney, D. (2007). Lateral inferotemporal cortex maintains conceptual-semantic representations in verbal working memory. Journal of Cognitive Neuroscience, 19, 2035-2049.

Fowler, A. E (1991). How early phonological development might set the stage for phonological awareness. In S. Brady \& D. P. Shankweiler (Eds.), Phonological processes in literacy: A tribute to Isabelle Y. Liberman (pp. 97-117). Hillsdale, NJ: Lawrence Erlbaum.

Gathercole, S. E. (1995). Is nonword repetition a test of phonological memory or long-term knowledge? It all depends on the nonwords. Memory \& Cognition, 23, 83-94. 
Gathercole, S. E., \& Baddeley, A. D. (1989). Evaluation of the role of phonological STM in the development of vocabulary in children: A longitudinal study. Journal of Memory and Language, 28, 200-213.

Gathercole, S. E., Frankish, C. R., Pickering, S. J., \& Peaker, S. (1999). Phonotactic influences on short-term memory. Journal of Experimental Psychology: Human Learning and Memory, 25, 84-95.

Gathercole, S. E., Hitch, G. J., Service, E., \& Martin, A. J. (1997). Phonological short-term memory and new word learning in children. Developmental Psychology, 33, 966-979.

Gathercole, S. E., Willis, C. S., Baddeley, A. D., \& Emslie, H. (1994). The Children's Test of Nonword Repetition: A test of phonological working memory. Memory, 2, 103-127.

Gathercole, S. E., Willis, C. S., Emslie, H., \& Baddeley, A. D. (1992). Phonological memory and vocabulary development during the early school years: A longitudinal study. Developmental Psychology, 28, 887-898.

Gupta, P. (2006). Nonword repetition, phonological storage, and multiple determination. Applied Psycholinguistics, 27, 564-568.

Henson, R. N. A. (1998). Short-term memory for serial order: The start-end model. Cognitive Psychology, 36, 73-137.

Hulme, C., Maughan, S., \& Brown, G. D. (1991). Memory for familiar and unfamiliar words: Evidence for a long-term memory contribution to short-term memory span. Journal of Memory and Language, 30, 685-701.

Kaufman, L., Vogel, S. E., Starke, M., Kremser, C., \& Schocke, M. (2009). Numerical and non-numerical ordinality processing in children with and without developmental dyscalculia: Evidence from fMRI. Cognitive Development, 24, 486-494.

Leclercq, A. L., \& Majerus, S. (2010). Serial order short-term memory predicts vocabulary development: Evidence from a longitudinal study. Developmental Psychology, 46, 417-427.

Lee, C. L., \& Estes, W. K. (1981). Item and order information in short-term memory: Evidence for multilevel perturbation processes. Journal of Experimental Psychology: Human Learning and Memory, 7, 149-169.

Majerus, S., Attout, L., D’Argembeau, A., Degueldre, C., Fias, W., Maquet, P., et al (2012). Attention supports verbal short-term memory via competition between dorsal and ventral attention networks. Cerebral Cortex, 22, 1086-1097.

Majerus, S., \& D’Argembeau, A. (2011). Verbal short-term memory reflects the organization of long-term memory. Further evidence from short-term memory for emotional words. Journal of Memory and Language, 64, 181-197.

Majerus, S., D’Argembeau, A., Martinez, T., Belayachi, S., Van der Linden, M., Collette, F., et al (2010). The commonality of neural networks for verbal and visual short-term memory. Journal of Cognitive Neuroscience, 22, 2570-2593.

Majerus, S., Heiligenstein, L., Gautherot, N., Poncelet, M., \& Van der Linden, M. (2009). The impact of auditory selective attention on verbal short-term memory and vocabulary development. Journal of Experimental Child Psychology, 103, 66-86.

Majerus, S., Norris, D., \& Patterson, K. (2007). What do patients with semantic dementia remember in verbal short-term memory? Sounds and order but not words. Cognitive Neuropsychology, 24, 131-151.

Majerus, S., Poncelet, M., Elsen, B., \& Van der Linden, M. (2006). Exploring the relationship between new word learning and short-term memory for serial order recall, item recall, and item recognition. European Journal of Cognitive Psychology, 18, 848-873.

Majerus, S., Poncelet, M., Greffe, C., \& Van der Linden, M. (2006). Relations between vocabulary development and verbal shortterm memory: The importance of short-term memory for serial order information. Journal of Experimental Child Psychology, 93, 95-119.

Majerus, S., Poncelet, M., Van der Linden, M., Albouy, G., Salmon, E., Sterpenich, V., et al (2006). The left intraparietal sulcus and verbal short-term memory: Focus of attention or serial order? NeuroImage, 32, 880-891.

Majerus, S., Poncelet, M., Van der Linden, M., \& Weekes, B. (2008). Lexical learning in bilingual adults: The relative importance of short-term memory for serial order and phonological knowledge. Cognition, 107, 395-419.

Majerus, S., \& Van der Linden, M. (2003). The development of long-term memory effects on verbal short-term memory: A replication study. British Journal of Developmental Psychology, 21, 303-310.

Majerus, S., Van der Linden, M., Mulder, L., Meulemans, T., \& Peters, F. (2004). Verbal short-term memory reflects the sublexical organization of the phonological language network: Evidence from an incidental phonotactic learning paradigm. Journal of Memory and Language, 51, 297-306.

Marshuetz, C., Smith, E. E., Jonides, J., DeGutis, J., \& Chenevert, T. L. (2000). Order information in working memory: fMRI evidence for parietal and prefrontal mechanisms. Journal of Cognitive Neuroscience, 12, 130-144.

Martin, R. C., Lesch, M. F., \& Bartha, M. C. (1999). Independence of input and output phonology in word processing and shortterm memory. Journal of Memory and Language, 41, 3-29.

Martinez Perez, T., Majerus, S., \& Poncelet, M. (2012a). The contribution of short-term memory for serial order to early reading acquisition: Evidence from a longitudinal study. Journal of Experimental Child Psychology, 111, 708-723.

Martinez Perez, T., Majerus, S., \& Poncelet, M. (2012b). Evidence for a specific impairment of serial order short-term memory in dyslexic children. Dyslexia, 18, 94-109.

Martinez Perez, T., Majerus, S., \& Poncelet, M. (2013). Impaired short-term memory for order in adults with dyslexia. Research in Developmental Disabilities, 34, 2211-2223.

McCormack, T., Brown, G. D. A., Vousden, J. I., \& Henson, R. N. A. (2000). Children's serial recall errors: Implications for theories of short-term memory development. Journal of Experimental Child Psychology, 76, 222-252.

Metsala, J. L. (1999). Young children's phonological awareness and nonword repetition as a function of vocabulary development. Journal of Educational Psychology, 91, 3-19.

Nairne, J. S., \& Kelley, M. R. (2004). Separating item and order information through process dissociation. Journal of Memory and Language, 50, 113-133.

Page, M., \& Norris, D. (2009). Is there a common mechanism underlying word-form learning and the Hebb repetition effect?: Experimental data and a modelling framework. In A. Thorn \& M. Page (Eds.), Interactions between short-term and long-term memory in the verbal domain (pp. 136-156). Hove, UK: Taylor \& Francis.

Papagno, C., Valentine, T., \& Baddeley, A. D. (1991). Phonological short-term memory and foreign-language vocabulary learning. Journal of Memory and Language, 30, 331-347.

Poirier, M., \& Saint-Aubin, J. (1996). Immediate serial recall, word frequency, item identity, and item position. Canadian Journal of Experimental Psychology, 50, 408-412.

Raven, J. C., Court, J. H., \& Raven, J. (1998). Progressive matrices couleur. Oxford, UK: Oxford Psychologists Press. 
Saint-Aubin, J., \& Poirier, M. (2005). Word frequency effects in immediate serial recall: Item familiarity and item co-occurrence have the same effect. Memory, 13, 325-332.

Schweickert, R. (1993). A multinomial processing tree model for degradation and redintegration in immediate recall. Memory E Cognition, 21, 168-175.

Service, E. (1992). Phonology, working memory, and foreign-language learning. Quarterly Journal of Experimental Psychology: Human Experimental Psychology A, 45, 21-50.

Szmalec, A., Duyck, W., Vandierendonck, A., Mata, A. B., \& Page, M. P. A. (2009). The Hebb repetition effect as a laboratory analogue of novel word learning. Quarterly Journal of Experimental Psychology, 62, 435-443.

Szmalec, A., Loncke, M., Page, M., \& Duyck, W. (2011). Order or disorder? Impaired Hebb learning in dyslexia. Journal of Experimental Psychology: Learning, Memory, and Cognition, 37, 1270-1279.

Tabachnick, B. G., \& Fidell, L. S. (1996). Using multivariate statistics (3rd ed.). New York: HarperCollins.

Thompson, B. (1993). The use of statistical significance tests in research: Bootstrap and other alternatives. Journal of Experimental Education, 61, 361-377.

Thorn, A. S., \& Frankish, C. R. (2005). Long-term knowledge effects on serial recall of nonwords are not exclusively lexical. Journal of Experimental Psychology: Learning, Memory, and Cognition, 31, 729-735.

Tubach, J. L., \& Boë, L. J. (1990). Un corpus de transcription phonétique. Paris: Telecom.

Walker, I., \& Hulme, C. (1999). Concrete words are easier to recall than abstract words: Evidence for a semantic contribution to short-term serial recall. Journal of Experimental Psychology: Learning, Memory, and Cognition, 25, 1256-1271. 